\title{
Alvar Núñez Cabeza de Vaca oder der fortgesetzte Schiffbruch
}

Doch Hans Staden war keineswegs ein Einzelfall, sondern steht mit seiner Person für eine Vielzahl von Europäern, die zwischen Alter und Neuer Welt im 16. Jahrhundert pendelten und letztlich das Kolonialsystem mit seinem kolonialen Räderwerk in Gang hielten. Denn es waren nicht nur die großen Heerführer oder Kapitäne, sondern vor allem die Söldner und Soldaten Spaniens und Europas, die Matrosen und Wachtposten, die Hafen- oder Zollbeamte, die Packer und Entlader diesseits und jenseits des Atlantik, die in ihren jeweiligen Funktionen das gigantische System des transatlantischen Kolonialismus zusammenhielten.

Auf dem weiten Feld der Reiseberichte habe ich mich daher für ein weiteres Beispiel sozusagen aus der zweiten Reihe der einfachen Soldaten entschieden. Und für ein Beispiel, welches erneut die Gefährlichkeit der Aufgaben innerhalb des kolonialen Abenteuers der 'Großen' gerade auch für die kleinen Leute demonstriert. Ein neben Hans Staden weiteres eindrucksvolles Exempel für einen Schiffbruch mit Überlebenden bietet Alvar Núnez Cabeza de Vaca, der an der Expedition des Pánfilo de Narváez teilnahm. Diese Expedition startete im Juni 1527 vom Hafen von Sanlúcar de Barrameda aus - wo sich später auch Staden zu seiner zweiten Reise nach Amerika einschiffen sollte -, um sich (anders als etwa Hernán Cortés, den Pánfilo de Narváez noch an seinem illegalen Vordringen ins Zentrum des Aztekenreiches hatte hindern wollen) mit rechtlicher Billigung des Kaisers Karl V alias Carlos I an die Eroberung des 1512 von Europäern 'entdeckten' Florida zu machen.

Erinnern wir uns bei dieser Gelegenheit daran, dass es sich bei diesen Expeditionen gleichsam um frühkapitalistische Unternehmungen handelte, die mit staatlicher Genehmigung ein Kapital zur Eroberung bestimmter Territorien aufbrachten, die dann nicht zuletzt für den privaten Gewinn der Kapitalgeber ausgebeutet werden konnten. Das war äußerst lukrativ und rentabel, aber durchaus auch riskant. Die gemeinen Soldaten hatten davon aber rein gar nichts, abgesehen von ihrer bezahlten Arbeit, für die sie freilich nur so lange bezahlt wurden, wie der Unternehmer die Möglichkeiten dazu hatte. Es war folglich ein frühkapitalistisches System sozusagen mit venture capital: alles andere als risikolos, aber höchst effizient in der Ausplünderung eigentlich fremder Territorien.

Nun, die Expedition des glücklosen Narváez geriet - anders als der Siegeszug des ebenso entschlossenen wie durchtriebenen Hernán Cortés - rasch zur Katastrophe. Bald schon wurde das aus rund sechshundert Mann bestehende 


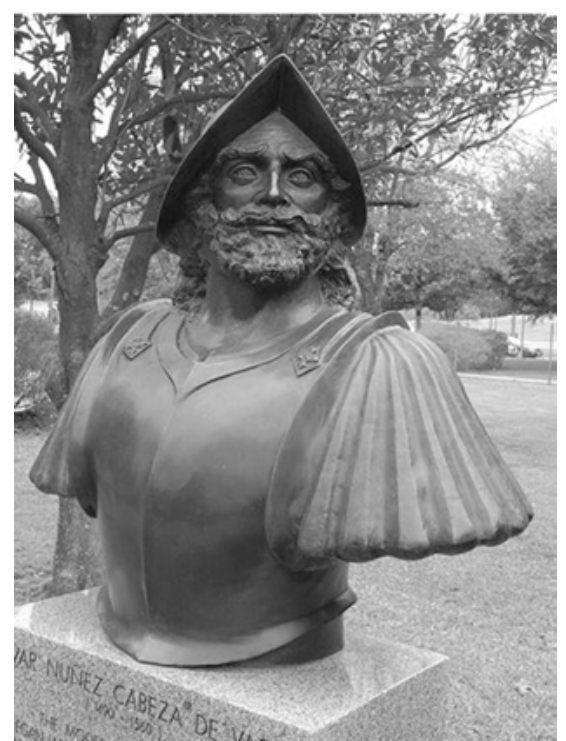

Abb. 45: Alvar Nuñez Cabeza de Vaca (Jerez de la Frontera, um 1490 - Sevilla, 1557).

Heer der Spanier durch Schiffbruch, Krankheiten und die ständigen Angriffe nomadisierender Indianer so stark dezimiert und in alle Winde zerstreut, dass von der ursprünglichen Armada nur noch ganze vier Männer übrig blieben unter ihnen Alvar Núnez Cabeza de Vaca. ${ }^{1}$ Und auch das gehörte zum Risiko des Unternehmers: Von Pánfilo de Narváez hat niemals mehr jemand gehört.

Die Katastrophe dieser Expedition, die für die unabhängigen SemiolenIndianer von Florida ein Gücksfall war, bestand in der Havarie der Schiffe und im eigentlichen Schifffbruch. Auch Alvar Núñez Cabeza de Vaca überlebte schließlich diesen Schiffbruch - oder eine ganze Abfolge von Schiffbrüchen der Expedition und stellte dies mit bewegenden Worten dar. Denn es waren Schiffbrüche mit Überlebenden: Nicht alle Schiffe gingen mit Mann und Maus unter. Und wie bei Hans Staden bildete auch bei diesem wackeren Spanier ein Schiffbruch den Ausgangspunkt für das eigentliche 'Abenteuer', von welchem uns sein Reisebericht auf zugegebenermaßen spannende Weise erzählt.

Kein anderer Titel hätte Cabeza de Vacas Bericht nachträglich besser auf den Punkt bringen können als jener der Naufragios, der Schiffbrüche, die sich

1 Vgl. hierzu sowie zu den reiseliterarischen Bewegungsfiguren seines Berichts ausführlicher Ette, Ottmar: Literatur in Bewegung. Raum und Dynamik grenzüberschreitenden Schreibens in Europa und Amerika. Weilerswist: Velbrück Wissenschaft 2001, S. 92-97. 


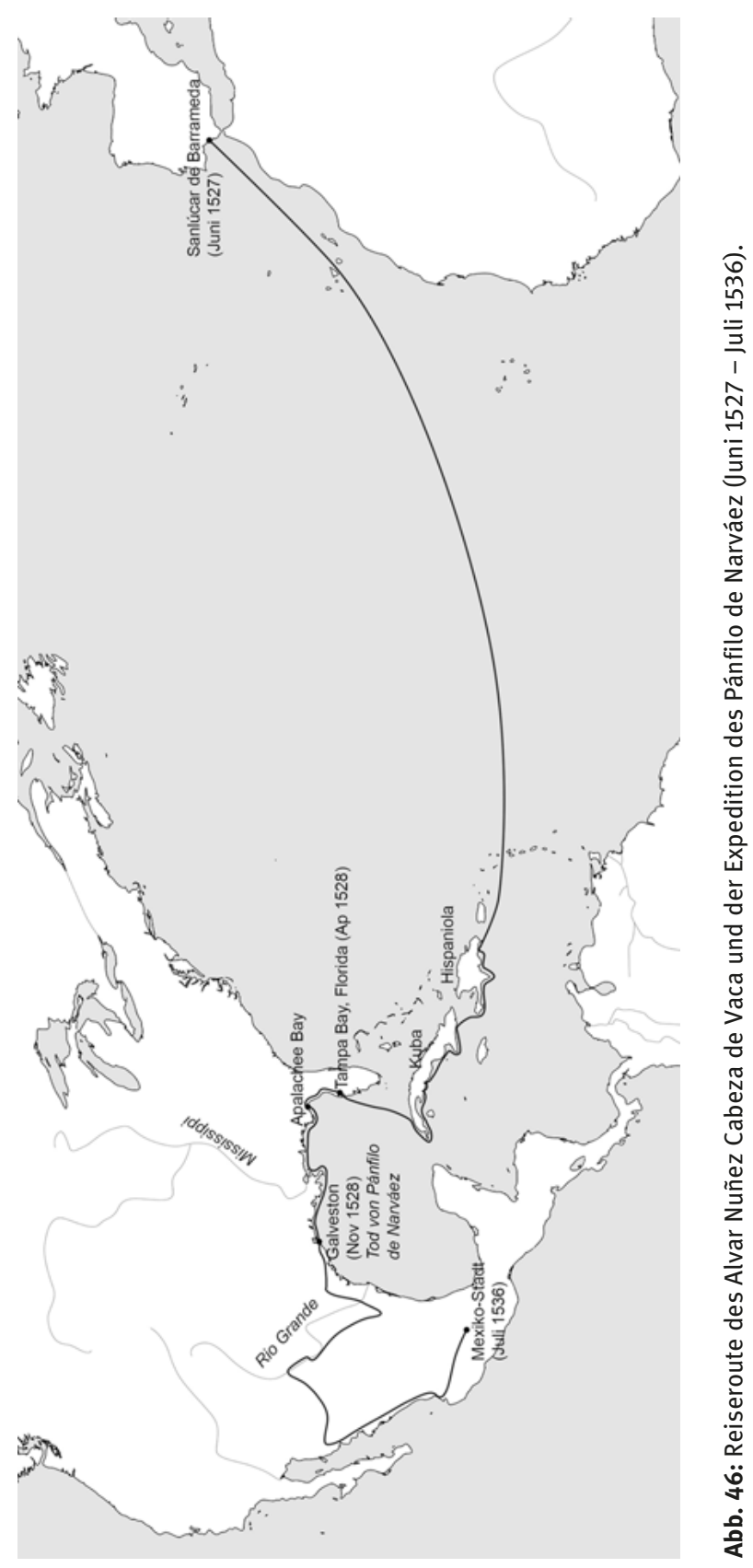


zwischen 1537 und 1540 ereigneten. ${ }^{2}$ Sie lagen erstmals im Jahre 1542 veröffentlicht vor. ${ }^{3}$ Bis dahin aber war es für ihren Verfasser buchstäblich ein weiter Weg gewesen, den wir hier anhand weniger Passagen nachvollziehen wollen.

Denn es handelt sich um den Bericht eines einfachen Soldaten, der in der ersten Phase beschleunigter Globalisierung plötzlich all seine Sicherheit verliert, seiner angestammten Rolle als glanzvoller spanischer Conquistador verlustig geht, einer ihm völlig fremden Umgebung schutzlos preisgegeben ist und $\mathrm{zu}$ einem wahren Überlebenskünstler avanciert. Er wird von einer einzigen Idee beseelt sein: Sich nur irgendwie wieder in den Bereich spanischer Herrschaft durchzuschlagen. Irgendwie das ganze Unglück ein für alle Mal hinter sich zu lassen.

Wie der protestantische Deutsche Hans Staden hat der katholische Spanier Alvar Núñez Cabeza de Vaca sein Gottvertrauen nicht verloren, ist aber (wie der Verfasser der Warhaftigen Historia in Brasilien) vor allem darauf angewiesen, das Leben der nomadisierenden Indianer im östlichen, mittleren und westlichen Teil der heutigen USA zu begreifen. Wieder also haben wir es hier mit der simplen Frage zu tun, wieso gerade dieser einfache Soldat überleben konnte. Nun, die Antwort - aber nicht deren Ausführung - ist relativ einfach: Weil er es verstand, die indigenen Gesellschaften, mit denen er in Kontakt stand, so $z u$ verstehen, dass er daraus ein für ihn wichtiges Lebenswissen und Überlebenswissen ableiten konnte. Denn nur durch ein solches Verstehen konnte er all jene interkulturellen Missverständnisse vermeiden, die für ihn zweifellos fatale Folgen gehabt hätten. Auch Alvar Núñez Cabeza de Vaca wurde damit zu einem sagen wir es etwas altmodisch - Völkerkundler avant la lettre.

Für diesen Prozess verfügte er über viel Zeit, über sehr viel Zeit sogar. Um es gleich vorwegzunehmen: In einem unvorstellbar mühevollen und oft monatelang unterbrochenen Marsch wird er den gesamten Süden Nordamerikas von den Küsten Floridas bis zu den Küsten Kaliforniens durchqueren, bevor es ihm gelingt, sich mit seinen verbliebenen Gefährten in das von Cortés eroberte Neuspanien und zu dessen Hauptstadt México durchzuschlagen, wo man die Gruppe 1535 - gleichsam im Zentrum eines gerade erst globalisierten Raumes triumphal empfängt. Kein Wunder: Jeder der dort lebenden Europäer konnte sich leibhaftig vorstellen, was diese Gruppe mitgemacht haben musste und war verblüfft, dass sie alle trotz eines derart langen, jahrelangen Marsches ihr Ziel

2 Vgl. hierzu Ferrando, Roberto: Introducción. In: Núñez Cabeza de Vaca, Alvar: Naufragios y Comentarios. Edición de Roberto Ferrando. Madrid: Historia 16 1984, S. 27 f.

3 Der Titel der erstmals im Oktober 1542 veröffentlichten Naufragios lautet: Relación que dió Alvar Núnez Cabeza de Vaca de lo acaescido en las Indias en la armada donde iva por governador Pánfilo de Narvaez [. . .]. 
niemals aus den Augen gelassen hatten. Und dass sie es schließlich geschafft hatten, sich quer durch indianisches Feindesland nach Neuspanien durchzuschlagen.

Wie Hans Stadens Bericht waren auch die Naufragios von unschätzbarem ethnographischem Wert, um die Lebensbedingungen der unterschiedlichen und in der Folge ausgelöschten indianischen Völker und Kulturen in dem von Núñez Cabeza de Vaca durchquerten und mit literarischen Mitteln dargestellten Raum rekonstruieren zu können. Wir verfügen letztlich über keine anderen Quellen, die gleichsam 'von innen' die indigenen Gemeinschaften beschreiben und all jenen Artefakten Leben geben, welche heute nur noch als 'tote' Ausgrabungsstätten im Süden der USA vor unseren Augen liegen. Ja, es war ein Bericht, dessen Wahrheitsgehalt wiederholt angezweifelt wurde; aber immer wieder haben Forschungen gezeigt, wie nahe Alvar Núñez Cabeza de Vaca bei der Wahrheit blieb und wie getreu er all das wiedergab, was er gesehen und vor allem intensiv erlebt hatte. Dass er dafür auch nach literarischen Formen und Vorbildern suchte, die seiner Zeit entstammten, ist angesichts des grundlegend friktionalen Status jedes Reiseberichts ganz selbstverständlich.

Die eher ethnographisch ausgerichteten Beobachtungen stehen über weite Strecken des Reiseberichts aber immer wieder im Vordergrund der Schilderungen von Alvar Núñez Cabeza de Vaca. Es verwundert daher nicht, dass in die narrativen Grundstrukturen seines Berichts immer wieder längere beschreibende Ausführungen eingelassen sind, in denen die Lebensformen der Indianer und ihr Gemeinschaftsleben dargestellt werden. Dabei betont der Spanier dankbar, dass die Indianer den Überlebenden der Expedition zunächst mit Lebensmitteln viel Fisch und vor allem „unos raíces que ellos comen, y son como nueces, algunas mayores o menores“4 - aushalfen. Dies ist ein Verhalten, das wir auch bei anderen Gelegenheiten bei den sehr unterschiedlichen Stämmen in Nordamerika beobachten können und das auch die legendären Pilgrim Fathers vor dem Hungertod bewahrte. Es belegt erneut, wie unvoreingenommen die indigenen Gruppen anderskulturellen Fremdlingen begegneten und mit diesen ihre gewiss knappen Lebensmittelvorräte teilten. Alvar Núñez Cabeza de Vaca jedenfalls war gegenüber diesen indigenen Gruppen von größtem Dank erfüllt.

Von Beginn seines Reiseberichtes der Naufragios an schilderte der Spanier stets aufmerksam die Interaktionsformen mit den Indianern, die - wie der Ich-Erzähler glaubt - durch ihr Weinen großes Mitleid mit dem Häuflein versprengter Spanier bekundeten. Hier lag freilich durchaus ein interkulturelles Missverständnis zu Grunde, welches jedoch keine fatalen Folgen zeitigte. Denn auf Seiten der Indigenen handelte es sich aller Wahrscheinlichkeit nach um

4 Núñez Cabeza de Vaca, Alvar: Naufragios y Comentarios, S. 71. 
einen Ritus, welcher keineswegs dem individuellen Schicksal der Überlebenden des Schiffbruchs galt.

Auch wenn es sich bei den „indios“ aus Sicht des Erzählers um „hombres tan sin razón y tan crudos, a manera de brutos ${ }^{* 5}$ handelt, werden doch bei aller Distanz die Lebensformen und die Lebensnormen der Indianer so genau beobachtet, dass hieraus ein Überlebenswissen und auch ein ZusammenLebensWissen gewonnen werden konnte, das in den Naufragios später literarisch festgehalten wurde. Auch in diesem Bericht steht das Zusammenleben der Indianer immer wieder im Vordergrund, wobei nicht nur wie bei Hans Staden ein besonderes Augenmerk den Beziehungen zwischen Männern und Frauen geschenkt wird, sondern gerade auch Konfliktlösungsstrategien bei Auseinandersetzungen von großem Interesse sind:

[...] y cuando en algunos pueblos riñen y traban cuestiones unos con otros, apuñéanse y apaléanse hasta que están cansados, y entonces se desparten; algunas veces los desparten mujeres, entrando entre ellos, que hombres no entran a despartirlos; y por ninguna pasión que tengan no meten en ella arcos ni flechas; y desque se han apuñeado y pasado su cuestión, toman sus casas y mujeres, y vanse a vivir por los campos y apartados de los otros, hasta que se les pasa el enojo; y cuando ya están desenojados y sin ira, tórnanse a su pueblo, y de ahí adelante son amigos como si ninguna cosa hobiera pasado entre ellos, ni es menester que nadie haga las amistades, porque de esta manera se hacen $[\ldots .]^{6}$

Derlei Beobachtungen sind für den einfachen Spanier von größter Wichtigkeit, beginnt er doch zu verstehen, auf welchen Grundlagen sich jene Formen und auch Normen entwickelten, die gerade auch die Hilfe und das zeitweilige Zusammenleben der Indianer mit den Spaniern ermöglichten. Es handelt sich so um letztlich überlebenswichtige Beschreibungen und Erkenntnisse.

Dabei wurden alle Formen des gemeinschaftlichen Zusammenlebens vorsichtig ausgeleuchtet. Auch Grausamkeiten und Morde unter den Indianern wurden während der langen Jahre ständigen Kontakts mit immer wieder wechselnden indianischen Gruppen genau registriert, wobei die cristianos - auch hier ist wie bei Staden die Religionszugehörigkeit das entscheidende distinktive Merkmal - selbst auch Funktionen für die indigenen Gemeinschaften übernahmen und etwa mit der Heilung von Kranken beauftragt wurden. So ergibt sich zwischen indigener Gemeinschaft und Spaniern ein symbiotisches Verhältnis oder doch zumindest eines, das man zumindest als arbeitsteilig ansprechen könnte. wie bei Hans Staden ist diese Übertragung von Funktionen auf die Fremden, die 'Zugereisten', von

5 Ebda., S. 73.

6 Ebda., S. 104. 
einer grundlegenden, vielleicht sogar lebensrettenden Bedeutung. Auch die genderspezifische Dimension spielt hierbei eine wichtige Rolle.

Das Zusammenleben mit wechselnden indigenen Gruppen erstreckt sich auch auf spirituelle und medizinische Bereiche. Nachdem sich einige erkrankte Indianer nach einer eher rituellen Behandlung durch die Christen - „santiguado y encomendado a Dios“" - als geheilt empfanden, beginnen auch hier Austauschprozesse zwischen indios und cristianos, insofern letztere zumindest vorübergehend auch in die (symbolische) Ökonomie indigener Gemeinschaften eingebaut werden. Auch dies ist ohne jeden Zweifel ein für die Spanier lebensrettender Aspekt einer fast schon symbiotisch $\mathrm{zu}$ nennenden Konvivenz. Die spanischen Schiffbruchsüberlebenden bieten Dienste an, die ein - wie man im heutigen Bürokratendeutsch sagen würde - Alleinstellungsmerkmal aufweisen und von den Indianern geschätzt werden.

Anders als andere Versprengte oder Schiffbrüchige, die in den indigenen Gruppen blieben und nicht mehr zu den 'Christen' zurückkehrten, gaben weder Hans Staden noch Alvar Núñez Cabeza de Vaca ihre Hoffnung auf, einen Weg zurück zu den Europäern und in ihre Heimat zu finden. Gerade für den Spanier, der freilich keine konkrete Vorstellung von den tatsächlichen Distanzen zwischen dem heutigen Süden der USA und der neuspanischen Hauptstadt hatte, wurde die Rückkehr zu einer fast obsessiven Vorstellung. Und es gelang ihm, diese Vorstellung nach langen, entbehrungsreichen Jahren in die Tat umzusetzen und nicht bei den indigenen Gemeinschaften zu bleiben. Dazu hatte mehrfach die Möglichkeit bestanden; aber dann hätten wir niemals etwas von diesen Naufragios gehört und die Mannschaft des Pánfilo Narváez einfach abgeschrieben.

So gibt es viele Parallelen zwischen den Reiseberichten eines Hans Staden und eines Alvar Núñez Cabeza de Vaca. Beider Berichte entwerfen globalisierte Lebensläufe, in denen - zumindest temporär - nicht nur interkulturelle, sondern auch transkulturelle Verhaltensweisen und Prozesse deutlich erkennbar werden. Diese transkulturellen Dimensionen, die Übernahme anderskultureller Praktiken in die eigenen, waren für beide Soldaten zweifellos überlebenswichtig.

Die Naufragios des Alvar Núñez Cabeza de Vaca stießen wie die Historia des Hans Staden auf ein riesiges Interesse beim zeitgenössischen Lesepublikum ebenso in Spanien wie im deutschsprachigen Raum. Die große Popularität derartiger Berichte von Schiffbrüchen mit Überlebenden verdankt sich - wie mir scheint - nicht nur einem gewissen Sensationsbedürfnis eines oftmals weit gestreuten europäischen Publikums oder der Vertrautheit breiter Leserschichten mit literarischen Formen, die vom mittelalterlichen Ritter- und Abenteuerroman

7 Ebda., S. 95. 
bis zum frühneuzeitlichen Schelmenroman reichen und das geneigte Lesepublikum auf derartige Berichte entsprechend vorbereiten konnten. Vielmehr entwerfen diese Berichte friktionale (also zwischen Diktion und Fiktion ${ }^{8}$ oszillierende) Erfahrungsräume, die vor dem globalisierten Horizont des 16. Jahrhunderts den Schiffbruch mit Zuschauer ${ }^{9}$ als konkrete Daseinsmetapher von Überlebenden präsentieren, welche sich als aktive Mitglieder einer weltweit expandierenden europäischen Gesellschaft plötzlich unter gänzlich anderen Lebens- und Überlebensbedingungen wiederfinden können. Angesichts der konkreten Zahlen außerhalb Europas lebender Europäer, die innerhalb des globalen kolonialen Räderwerks ihr Auskommen gefunden hatten, ist eine derartige Vertrautheit der Daheimgebliebenen mit 'nahen' Schicksalen von 'Weggegangenen' sehr wohl gegeben.

Wie sehr sich das Lokale und das Globale längst durchdringen konnten, mag das nur auf den ersten Blick überraschende Ende der Naufragios belegen, mit dem die vier Überlebenden des Schiffbruchs der Armada von Pánfilo de Narváez dem Lesepublikum vorgestellt werden:

Y pues he dado relación de los navíos, será bien que diga quién son y de qué lugar de estos reinos, los que nuestro Señor fue servido de escapar de estos trabajos. El primero es Alonso del Castillo Maldonado, natural de Salamanca, hijo del doctor Castillo y de doña Aldonza Maldonado. El segundo es Andrés Dorantes, hijo de Pablo Dorante, natural de Béjar y vecino de Gibraleón. El tercero es Alvar Núñez Cabeza de Vaca, hijo de Francisco de Vera y nieto de Pedro de Vera, el que ganó a Canaria, y su madre se llamaba doña Teresa Cabeza de Vaca, natural de Jerez de la Frontera. El cuarto se llama Estebanico; es negro alárabe, natural de Azamor. ${ }^{10}$

In diesen wenigen Zeilen werden nicht nur vier Schicksale individueller Menschen zusammengefasst, die sich ursprünglich einmal der Gewinn versprechenden Expedition des Pánfilo de Narváez angeschlossen hatten und sich zweifellos vom Geschäft mit den Indias große persönliche Vorteile versprachen. Sie alle bestiegen die Schiffe, um in der kolonialen empresa, in der kolonialen Unternehmung ihres Anführers, Vorteile für sich herauszuschlagen. Vielmehr wird eine ganze Welt, die Welt der ersten Phase beschleunigter Globalisierung, mit Händen greifbar und an Einzelschicksalen vorgeführt.

8 Vgl. zum gattungsspezifischen Hintergrund der Naufragios u. a. Maura, Juan Francisco: Introducción. In: Núñez Cabeza de Vaca, Alvar: Naufragios. Edición de Juan Francisco Maura. Madrid: Ediciones Cátedra 2000, S. 35-41.

9 Vgl. hierzu Blumenberg, Hans: Schiffbruch mit Zuschauer. Paradigma einer Daseinsmetapher. Frankfurt am Main: Suhrkamp 1979.

10 Núñez Cabeza de Vaca, Alvar: Naufragios y Comentarios, S. 142 (der Druckfehler „Estabanico“ wurde hier getilgt). 
Die in dieser Schlußpassage der Naufragios aufgerufenen Orte - von der ehemaligen Grenzstadt zum Nasridenreich Jerez bis hin zur erst vor kurzem eroberten Hauptinsel der Kanaren -, nicht zuletzt aber auch der negro alárabe machen uns darauf aufmerksam, mit welcher Gewalt und Geschwindigkeit in dieser Phase beschleunigter Globalisierung Europa, Afrika und Amerika, die Welt des Islam, der Guanchen, der amerikanischen Religionen und des Christentums hier aufeinander stoßen. Die Welt ist nur wenige Jahrzehnte nach der sogenannten 'Entdeckung' Amerikas zu einem wahren Wirbel an Bewegungen geworden, der sich noch immer stetig verstärkt. Die für diese Entwicklung geradezu emblematische Erwähnung von Estebanico wirkt ganz am Ende des Berichts wie ein Wasserzeichen der Globalisierung - und ein erstes Indiz für die so lange von der Forschung vernachlässigten arabamerikanischen Beziehungen. ${ }^{11}$

Doch ist auch dies nur ein weiteres Zeichen für eine weltumspannende Mobilität, für eine ungeheure Bewegung, in welcher menschliche Schicksale - angetrieben von immer neuen empresas - weltweiten Zuschnitt erfahren. Die von Pietro Martire d'Anghiera zu Beginn dieser Phase konstatierte und in einer vorangegangenen Vorlesung angeführte Bewegung des 'Auftrauchens' immer neuer Inseln am Horizont der europäischen Länder ist binnen weniger Jahrzehnte zu einem weltweiten Wirbel geworden. An den Einzelschicksalen dieser Überlebenden eines Schiffbruchs wird deutlich, was das Leben einer ganzen historischen Epoche ausmacht.

Doch vergessen wir nicht: Alle vier sind nur um Haaresbreite dem Schicksal der anderen sechshundert Männer, die aus Sanlúcar de Barrameda aufbrachen, um ihr Glück in der Neuen Welt zu versuchen, entgangen. Mag sein, dass sich einige dieser sechshundert Individuen in indigene Gemeinschaften retteten und dort bis zu ihrem Lebensende lebten. Die große Mehrzahl jedoch wird bei den Schiffbrüchen wie vor allem den sich anschließenden bewaffneten Konflikten mit der indigenen Bevölkerung zu Tode gekommen sein. Sie waren gleichsam die Betriebskosten der Empresa de Indias, die mit der ersten Fahrt des Columbus unwiderruflich begonnen hatte. Reisen und Schreiben stehen im Zeichen der Schiffbrüche in diesem Kontext in einem eigenartigen Verhältnis: in jenem des schieren Überlebens oder Überlebthabens.

Der Schiffbruch erscheint in dieser Konstellation wie die Kehrseite der Globalisierung: Wie die beiden Seiten ein und derselben Münze sind mondialisation und naufrage untrennbar miteinander verbunden. Die Naufragios wie die Warhaftige

11 Vgl. Ette, Ottmar / Pannewick, Friederike (Hg.): ArabAmericas. Literary Entanglements of the American Hemisphere and the Arab World. Frankfurt am Main - Madrid: Vervuert Verlag Iberoamericana 2006. 
Historia sind Zeugnisse vom Überlebenswillen wie vom ÜberLebenswissen in einer sich globalisierenden Welt, die jenseits der Kriegszüge, Massaker und Epidemien noch immer - die Figur des Las Casas zeigt dies ebenso eindringlich wie die späteren Debatten um die rechtliche Stellung der Indianer - nach dem Wissen für ein wie auch immer zu regelndes Zusammenleben zwischen Globalisierern und Globalisierten sucht. Denn im Aufeinanderprall verschiedenartigster Kulturen entsteht neben dem 'Gesetz des Dschungels' stets auch das Bedürfnis, das Zusammenleben neu zu ordnen und neue Formen für dieses Zusammenleben zu entwickeln.

In der Schiffbruch-Literatur werden Texte wie die hier stellvertretend behandelten zu Zeugnissen des eigenen Überlebens - und stehen zugleich für das Verstummen der Verschwundenen ein. Sie geben denjenigen eine Stimme, die über keine Stimme verfügten und von der kolonialen Maschinerie ausgespuckt wurden, ehe sie sich recht besonnen hatten. Wir sehen und erblicken in diesen Berichten von Schiffbrüchen nur einen Teil der Kosten der Globalisierung auf europäischer Seite. Fast mag man darüber vergessen, was an Gemetzeln, an Zerstörungen, an Massakern und an Genozid über die indigenen Bevölkerungen der Amerikas hereinbrach. Doch dass die weitere Ausbreitung der Europäer über die Welt in der völligen Katastrophe enden kann, ist in diesen Berichten und ihrem narrativ wie diskursiv in Szene gesetzten Wissen immer schon mitgedacht und sollte uns deutlich vor Augen stehen. 